Revista de Estudios Histórico-Jurídicos

[Sección historia del derecho patrio chileno]

XL (Valparaíso, Chile, 2018)

[pp. 371 - 390]

\title{
Moisés Poblete Troncoso y su aPORTE a la RECOPILACIÓN DE LA LEGISLACIÓN LABORAL
}

[Moisés Poblete Troncoso and your Contribution to the Compiling of Labor Legislation]

\author{
Juan Carlos Yáñez ANDRAdE* \\ Universidad de Valparaíso, Chile
}

\begin{abstract}
Resumen
El presente artículo destaca el trabajo de recopilación que hizo el abogado chileno Moisés Poblete Troncoso de la legislación laboral chilena y latinoamericana en el transcurso de los años 1920 y 1950 . Se presenta su trayectoria intelectual, su participación en la Oficina del Trabajo como Director (1920-1925), así como su recorrido internacional. En especial se destaca su paso por la Organización Internacional del Trabajo y su labor recopilatoria y de asistencia técnica que cumplió entre 1927 y 1937. Finalmente, se ofrecen aspectos que destacan los aportes concretos en materia de legislación laboral y de seguridad social.
\end{abstract}

Palabras clave

Moisés Poblete - Legislación laboral Seguridad social Chile - Legislación social América Latina.

\begin{abstract}
This article highlights the compilation work of the Chilean and Latin American labor legislation done by the Chilean lawyer Moises Poblete Troncoso during the 1920s and 1950s. His intellectual career, his participation in the Labor Office as Director (1920 -1925), as well as his international activity. In particular, it stands out for its work in the International Labor Organization and his compilation work and technical assistance that he carried out between 1927 and 1937. Finally, aspects that highlight the concrete contributions in terms of labor legislation and social security are offered.
\end{abstract}

\section{KeYwords}

Moisés Poblete - Labor legislation Social security - Chile - Latin America.

RECiBIDO el 28 de noviembre de 2017 y ACEPTADO el 3 de abril de 2018

* Doctor en Historia. Profesor de la Facultad de Ciencias Económicas y Administrativas de la Universidad de Valparaíso. Investigador CIDEP-UV. Dirección postal: 5 Oriente $n^{\circ} 180$, Viña del Mar. Correo electrónico: juancarlos.yanez@uv.cl 


\section{INTRODUCCIÓN}

El objetivo del presente artículo es destacar el trabajo de recopilación que hizo el abogado chileno Moisés Poblete Troncoso de la legislación laboral chilena y latinoamericana en el transcurso de los años 1920 y 1950. Moisés Poblete desarrolló su trabajo profesional en el momento mismo que eclosiona la cuestión social en Chile y se debaten las primeras propuestas legislativas para enfrentar el problema obrero. En este sentido, sus competencias intelectuales y el interés que muestra desde un comienzo por registrar los avances legislativos en materia social y laboral señalan el alto sentido de responsabilidad que tenía Poblete y su compromiso con los problemas de su tiempo.Un aspecto no menor fue su aporte a la historia laboral y su estudio sobre el movimiento obrero chileno y latinoamericano. En un trabajo de 1924 abordó la evolución de las organizaciones sindicales en Chile ${ }^{1}$. Posteriormente, en una publicación de 1946, y que lleva por título El movimiento obrero latinoamericano ${ }^{2}$, reconstruyó la formación y desarrollo sindical en distintos países, ofreciendo una periodificación de la evolución del movimiento obrero que tuvo amplia influencia en investigaciones posteriores.

En cuanto al reconocimiento de la obra de Poblete, existen pocos estudios sobre su itinerario intelectual. En particular se puede destacar el trabajo de Guillermo Feliú Cruz, donde, junto con abordar aspectos biográficos, da cuenta de su obra a través de un recuento bibliográfico ${ }^{3}$. Recientemente algunos autores han destacado su obra pionera acerca de la historia del movimiento obrero, pero sin enfocarse en su papel de recopilador de la legislación laboral de Chile y América Latina ${ }^{4}$.

El presente artículo se estructura en tres secciones. La primera, aborda -desde un punto de vista más bien descriptivo- el recorrido profesional de Poblete, desde su formación como abogado, pasando por sus primeras funciones en la administración pública, hasta el importante papel que cumplió como director de la Oficina del Trabajo y redactor del proyecto de Código Laboral presentado al Congreso Nacional en 1921 por el gobierno de Arturo Alessandri. En la segunda sección se destaca su labor realizada en la Organización Internacional del Trabajo (OIT) y su labor como redactor del primer informe sobre el estado de la legislación social en el continente. Este informe, no solo dio cuenta del nivel de avances en materia de legislación que presentaba el continente americano, sino también las vinculaciones que había logrado establecer la OIT con los países miembros. Por último, la sección final trata de la seguridad social en Chile y América, centrándose sobre cómo se instala la noción de seguridad social durante el periodo de estudio

1 Poblete, Moisés, La organización sindical en Chile y otros estudios sociales (Santiago, Imprenta Ramón Brias, 1926).

2 Poblete, Moisés, El movimiento obrero latinoamericano (México, FCE, 1946).

3 Feliú Cruz, Guillermo, Moisés Poblete Troncoso: la bibliografía de las ciencias sociales (Santiago, Biblioteca Nacional, 1970).

${ }^{4}$ Herrera, Patricio, La Confederación de Trabajadores de América Latina en la historiografía obrera. 1938-1963, en Cuadernos de Historia, 36 (Santiago, 2012), pp. 85-117; YÁÑEZ, Juan Carlos, Moisés Poblete Troncoso y la primera historia del movimiento obrero latinoamericano (1946), en Izquierdas, 37 (Santiago, 2017), pp. 261-280. 
y el trabajo de recopilación que hace Poblete sobre los niveles de protección social existente en los países a comienzos de la década de 1940.

En cuanto a fuentes y bibliografía consultadas se puede señalar el Archivo de la OIT, el cual se ubica en la ciudad de Ginebra y que ofrece aspectos curriculares de Poblete. Además, sus archivos contienen las tareas que le fueron asignadas durante el periodo y documentación sobre sus estudios recopilatorios. El acceso a la producción bibliográfica de su obra se encuentra en su mayoría en la Biblioteca Nacional y la Biblioteca del Congreso Nacional de Chile.

\section{EL RECORRIDO PROFESIONAL DE MOISÉS POBLETE TRONCOSO}

Moisés Poblete Troncoso se tituló de abogado con una memoria sobre legislación de hijos ilegítimos ${ }^{5}$, mostrando desde muy joven una preocupación por los problemas nacionales que afectaban al país, aunque sin involucrarse en la política contingente. En 1920 publicó un ensayo titulado El balance de nuestro seudo régimen parlamentario, criticando el sistema parlamentario chileno y mostrándose dispuesto a avanzar hacia una reforma política que reforzara el poder presidencial ${ }^{6}$.

Una vez electo Arturo Alessandri como presidente de la República, con una plataforma programática que incluía una serie de reformas sociales, legislación laboral, mayor intervención del Estado en los conflictos laborales y el reforzamiento de la institucionalidad, entre otros aspectos, Moisés Poblete fue nombrado director de la Oficina del Trabajo, institución que había aumentado sus atribuciones, pasando de ser una agencia de estadística a una de inspección laboral ${ }^{7}$. Su principal aporte fue avanzar en la institucionalización de la Oficinal del Trabajo y comprometer su acción en la defensa de los derechos de los trabajadores. Para ello intervino en la solución de algunas huelgas, apoyándose en el decreto de conciliación y arbitraje de 1917, y promovió conferencias de divulgación de la naciente legislación laboral. En 1921 apoyó la reforma legislativa promovida por el gobierno de Arturo Alessandri, elaborando el proyecto de Código Laboral que fue presentado al Congreso en junio de ese año.

El proyecto elaborado por Poblete incluía 600 artículos que comprendían temas muy diversos como las condiciones de trabajo, la organización sindical, los mecanismos de conciliación y arbitraje, la distribución de utilidades, entre otros. En materia de jornada laboral y condiciones de trabajo, el proyecto incorporaba los principios discutidos extensamente en el país y en la Conferencia Internacional del

\footnotetext{
5 Poblete, Moisés, Legislación sobre hijos ilegitimos (cuestión social) (Santiago, Imprenta El Progreso, 1912).

${ }^{6}$ Poblete, Moisés, El balance de nuestro seudo régimen parlamentario (Santiago, Talleres de Numen, 1920).

${ }^{7}$ La creación y funcionamiento de las primeras oficinas del trabajo, tanto en Chile como en el extranjero, ha tenido un gran interés en los últimos años. Véase, por ejemplo, YáñEz, Juan Carlos, La intervención social en Chile (1907-1932) (Santiago, Ril Editores, 2008); SuRIANO, Juan, El Departamento Nacional del Trabajo y la política laboral durante el primer gobierno de Hipólito Yrigoyen, en Ben Plotkin, Mariano y Zimmermann, Eduardo (compiladores), Los saberes del Estado (Buenos Aires, Edhasa, 2012).
} 
Trabajo de 1919 en Washington. Por ejemplo, se prohibía el trabajo de menores de 14 años, excluyendo aquellos ejercidos por los miembros de una familia y las actividades de las escuelas profesionales. Por otra parte, se prohibía el trabajo de jóvenes entre 14 y 18 años, que no hubiesen completado su instrucción primaria. Como aspecto importante, el proyecto prohibía el trabajo nocturno de mujeres y menores de 18 años, estableciendo, además, la jornada de 8 horas y semanal de 48 .

En cuanto a los sindicatos, se los entendía como una organización que reunía a empleados y obreros del mismo oficio o ramo, de manera voluntaria y reducida a la defensa de los intereses económicos de los asociados. Se limitaba su creación al sector privado, debiendo sus estatutos ser aprobados por la autoridad. Además, se proponía un sistema de conciliación y arbitraje voluntario para enfrentar los conflictos capital-trabajo, lo cual era un retroceso en cuanto a las necesidades de enfrentar el periodo de huelgas que el país atravesaba a partir de la crisis salitrera de 1919, estando en las antípodas del proyecto conservador que había declarado la conciliación y el arbitraje obligatorio ${ }^{8}$.

Un aspecto novedoso que consideraba el proyecto de Moisés Poblete era la creación de una Dirección General del Trabajo, cuyas funciones eran la compilación, coordinación y publicación de la información relativa al trabajo en diversas actividades productivas, especialmente la organización de la producción y las condiciones de los trabajadores. Además, podía estudiar y proponer al gobierno las medidas legales y administrativas con el fin de mejorar la condiciones material, moral e intelectual de los trabajadores. Por último, y quizás los más importante, la Dirección General del Trabajo tenía competencias fiscalizadoras en las empresas y toda actividad productiva. En el artículo 267 del proyecto, la Dirección General del Trabajo era divida en siete secciones que comprendían la legislación y publicaciones; la inspección y las estadísticas; los accidentes, la higiene y la seguridad del trabajo; la previsión social; y la asociación obrera; la sección internacional; la biblioteca y el intercambio; y por último el servicio de colocación obrera? ${ }^{9}$.

Las fuentes que usó Poblete para la elaboración de su proyecto son variadas y plenamente identificables. En primer lugar, envió un cuestionario a las organizaciones obreras para consultar su opinión sobre el proyecto, junto con demandar al Ministerio de Relaciones Exteriores el envío, a través de las embajadas, de la legislación laboral dictada en los diferentes países ${ }^{10}$. Si bien no conocemos si Poblete recogió en parte las opiniones de los trabajadores, este acto de consultarles a través de una encuesta refleja la personalidad siempre abierta y de buen trato hacia las organizaciones obreras que marcó su gestión en la dirección de la Oficina del Trabajo. Por otra parte, era claro que el proyecto retomaba las disposiciones que habían sido discutidas en la Conferencia del Trabajo de Washington en 1919 de la Organización Internacional del Trabajo (OIT). De esta manera, los argumentos principales para su aprobación provenían también desde ese contexto

\footnotetext{
${ }^{8}$ Morris, James, Las elites, los intelectuales y el consenso: estudio de la cuestión social y del sistema de relaciones laborales (Santiago, Editorial del Pacífico, 1967).

${ }^{9}$ Boletín de la Oficina del Trabajo, 17 (Santiago, 1921).

${ }^{10}$ Archivo Nacional, Dirección General del Trabajo, Vol. 76, Comunicaciones enviadas (Santiago, 1921).
} 
internacional. En 1921 y 1922, el presidente Arturo Alessandri, demandó al Congreso la rápida aprobación del proyecto, con el fin de cumplir con los acuerdos internacionales del país. El mismo Poblete comenzó a justificar la necesidad de aprobar la legislación social que estaba siendo discutida en el Congreso, como una manera de respetar esos acuerdos a los cuales Chile había adherido ${ }^{11}$.

Es en este periodo que nace su interés por la historia laboral chilena, ofreciendo los primeros aportes al conocimiento de ella. En 1924 publicó una recopilación de leyes junto a Óscar Álvarez Andrews, en la cual destaca la importancia de los primeros congresos internacionales promovidos por la OIT y las reuniones panamericanas en la difusión de nuevos principios sociales y laborales ${ }^{12}$. Poblete y Álvarez se mostraron críticos frente al pobre desarrollo de la legislación obrera, responsabilizando al Estado y las doctrinas liberales herederas del economista francés Courcelle-Seneuil por esta situación. Sin embargo, reconocían un cambio en la importancia que las autoridades le estaban dando al problema social en Chile, destacando al respecto el papel de las universidades y la promoción de los cursos de Economía Social.

El interés de Poblete por conectar sus preocupaciones sociales con las organizaciones internacionales comenzó a aparecer en esta época, lo que explica que se radicaran en la Oficina del Trabajo las relaciones con la OIT, desplazando con ello al Ministerio de Relaciones Exteriores. También se destaca su participación en la Conferencia Panamericana de 1923 como secretario de la delegación chilena, organizadora del encuentro. En esa calidad fue el responsable, a solicitud del político liberal Manuel Rivas Vicuña, de introducir una resolución para que en las futuras conferencias panamericanas se estudiaran las temáticas sociales ${ }^{13}$. En 1924 participó en el primer Congreso de Economía Social en la ciudad de Buenos Aires, con un estudio sobre la legislación social.

El desarrollo profesional de Moisés Poblete y su pertenencia a una red latinoamericana de intelectuales facilitó su vinculación con la OIT, ayudando a promover, por ejemplo, su revista de versión en habla hispana titulada Informaciones Sociales y apoyando los contactos entre Albert Thomas (director de la OIT) y diferentes personalidades políticas y sindicales de Brasil, Uruguay y Argentina ${ }^{14}$. Los informes que elaboró para la OIT, a propósito del programa del gobierno de Alessandri y los avances en la legislación laboral, dan cuenta no solo del cumplimiento de los compromisos asumidos por las autoridades chilenas en materia social, sino también de la cercanía, incluso amistad, que la relación epistolar fue generando entre Poblete y Thomas.

11 Ibíd.

12 Poblete, Moisés y Álvarez, Oscar, La legislación social obrera chilena (recopilación de leyes $y$ disposiciones vigentes sobre el trabajo y la previsión social) (Santiago, Imprenta Santiago, 1924).

13 Feliú Cruz, Guillermo, Moisés Poblete Troncoso: la bibliografía de las ciencias sociales (Santiago, Biblioteca Nacional, 1970), p. 11.

${ }^{14}$ Los informes de la visita del director de la OIT, Albert Thomas, a Chile, muestran los primeros contactos entre Poblete y los funcionarios de la organización de Ginebra. Al respecto véase YÁÑEz, Juan Carlos, La OIT en América del Sur. El comunismo y los trabajadores chilenos (1922-1932) (Santiago, Editorial UAH, 2016). 
La contratación de Moisés Poblete en 1927 como funcionario de la OIT se debió a circunstancias un poco casuales. En principio, el funcionario chileno se sentía incómodo por la situación política nacional y por la parálisis de la administración pública, en particular del recién creado Ministerio del Trabajo, Asistencia y Previsión Social (1925). Su carrera funcionaria parecía estancada y las posibilidades de crecimiento profesional no eran promisorias frente al aumento de poder de Carlos Ibáñez del Campo, quien había accedido a la Vicepresidencia de la República, por lo que este clima político deteriorado habría motivado a Poblete a solicitar un puesto en la OIT. Enseguida, la partida del único funcionario chileno que pertenecía a la institución de Ginebra, Carlos García Palacios, a la Secretaría de la Sociedad de las Naciones, dejó vacante un lugar que era necesario llenar, en lo posible, con otro funcionario sudamericano ${ }^{15}$. Valorado por su formación universitaria, sus responsabilidades administrativas y su cátedra en la Universidad de Chile, Poblete aceptó ser contratado de manera temporal, siendo incorporado a la División de Relaciones, en categoría de miembro de Sección B (con un sueldo de 1000 francos al mes). Sus funciones fueron las siguientes: a) ocuparse de las relaciones con Chile, b) dirigir la preparación de un volumen de la serie legislativa sobre la legislación social del continente, c) intensificar el esfuerzo de ratificación de los países sudamericanos ${ }^{16}$.

$\mathrm{Al}$ parecer razones presupuestarias hicieron imposible cumplir con los deseos de Albert Thomas de ofrecerle un puesto de miembro de Sección A, como estaba contemplado desde un comienzo. En todo caso, frente a los ofrecimientos que habría recibido Poblete de ocupar un empleo oficial en Europa o incluso Chile, Thomas consideró conveniente reglamentar su situación ofreciéndole un puesto permanente en la División de Informaciones y Relaciones ${ }^{17}$. Con ello, Poblete fue nombrado en 1929 funcionario permanente con un ingreso anual de 15.600 francos suizos.

Para Poblete, la incorporación a la OIT le significó acrecentar aún más sus competencias y transformarse rápidamente en un experto regional, debiendo, como una de sus primeras tareas, compilar la legislación social del continente americano. Este trabajo dio origen a un volumen doble titulado Legislación social de América Latina (1928-1929) ${ }^{18}$ con el cual se cumplió la exigencia reglamentaria que tenía la OIT de que al cabo de diez años de aprobados los primeros convenios se debía realizar un estudio sobre el estado de avance en su aplicación. En la época, Moisés Poblete Troncoso era una de las personalidades más importantes en lo que concierne el conocimiento de la legislación social del continente americano,

15 Herrera, Fabián y WeHrl, Yannick, Le Bureau international du Travail et l'Amérique latine durant l'entre-deux-guerres : problèmes et enjeux, en LESPINET-MORET, Isabelle y VIET, Vincent, L'Organisation internationale du Travail. Origine, développement et avenir (Rennes, PUR, 2011), p. 159.

${ }^{16}$ Véase el expediente personal de Poblete en los archivos de la OIT, Ginebra, Extrait du rapport du 3 mai 1927, $n^{\circ}$ 33, Archivo Organización Internacional del Trabajo [En adelante AOIT] P 2037, M. Poblete Troncoso.

17 Nota de Mario Viple, 16 de mayo de 1929, AOIT, P 2037, M. Poblete Troncoso.

18 OIT, La legislación social de América Latina, vol. 1-2, (Ginebra, OIT, 1928-1929). 
permitiéndole sus antiguas funciones como director de la Oficina del Trabajo tener un manejo directo de la gestión de los organismos de inspección laboral y entablar relaciones con las instituciones similares de otros países, así como con personalidades del mundo intelectual, político y sindical latinoamericano. De esta forma, cuando la OIT se propuso elaborar ese informe de la realidad legislativa de la región no había ningún otro profesional capaz de coordinar un esfuerzo de tal naturaleza. Si bien fue redactado en la ciudad de Ginebra durante 1927, la filiación con sus primeras publicaciones sobre el estado de la legislación social en Chile resulta evidente ${ }^{19}$. Además, este informe debe ser enmarcado en una perspectiva de largo plazo, siendo la base de una publicación del mismo Poblete de 1936, la cual reúne la bibliografía social de los países latinoamericanos, ofreciendo un verdadero catálogo actualizado de obras y autores sobre el tema ${ }^{20}$.

Todos estos trabajos de compilación de la legislación social le permitieron a Poblete dar un salto cualitativo al entablar nuevas relaciones con el medio latinoamericano, pensar la realidad continental desde un plano más global y ayudar en el esfuerzo de coordinación del trabajo legislativo que la OIT proyectaba para la primera década de su creación. Además, estos contactos, construidos en el curso de los años, fueron fundamentales al momento de utilizar esa red de amistades para comenzar a redactar su estudio titulado El movimiento obrero latinoamericano ${ }^{21}$.

Una vez instalado en Ginebra, y pese a las restricciones presupuestarias que impuso la crisis económica de 1929, Poblete viajó a Chile en 1930, donde realizó una serie de conferencias y tomó contactos con distintas autoridades, además de redactar informes sobre el gobierno del general Carlos Ibáñez del Campo. Recién en 1934 tuvo la posibilidad de participar en una nueva misión a latinoamericana, en el marco de los estudios y asesoría sobre las instituciones de previsión social y la reorganización del Ministerio del Trabajo de Cuba, donde recibió elogiosos comentarios por su labor. Sin embargo, fue la convocatoria a la Primera Conferencia Interamericana del Trabajo de 1936 la que permitió demostrar sus cualidades organizativas. Esta Conferencia se realizó por la invitación del gobierno de Chile durante el transcurso de la Conferencia Internacional del Trabajo de Ginebra en 1935, mostrándose interesado el gobierno de Arturo Alessandri (1932-1938) en consolidar sus relaciones con la OIT luego de la muerte de Albert Thomas en 1932. Por otra parte, el rol asignado a Poblete en la organización de esta Conferencia permite entender que con este evento se cerraba un primer ciclo de vinculaciones internacionales iniciado en 1920, con los primeros contactos entre el mismo Poblete, Arturo Alessandri y Albert Thomas, no siendo extraño que estas antiguas vinculaciones expliquen la disposición de la OIT a convocar una primera Conferencia Interamericana del Trabajo en la ciudad de Santiago de Chile. A propósito de esta Conferencia, Poblete publicó una investigación sobre los problemas sociales y económicos del continente, una suerte de balance sobre los progresos sociales de la región y que al mismo tiempo puede ser leído como

\footnotetext{
19 Poblete y Álvarez, cit. (n. 11).

${ }^{20}$ Poblete, Moisés, Ensayo de bibliografía social de los paises hispanoamericanos (Santiago,

${ }^{21}$ Poblete, Moisés, cit. (n. 2).
} La Nación, 1936). 
un escrito realizado por un intelectual de primera línea a fin de llamar la atención sobre las visiones prejuiciosas que la intelectualidad europea y norteamericana tenía sobre el continente, señalando claramente que los avances sociales en latinoamericana eran proporcionalmente superiores a aquellos de los países europeos ${ }^{22}$. De manera explícita destacaba una intelectualidad hispanoamericana preocupada por las cuestiones sociales y laborales, entre los cuales se pueden nombrar a los argentinos Juan Justo, Alfredo Palacios, Alejandro Unsain, Augusto Bunge, Tomás Amadeo, José Ingenieros y al mexicano Vicente Lombardo Toledano ${ }^{23}$.

Las resoluciones de la Conferencia Interamericana del Trabajo de 1936 tuvieron dos consecuencias concretas en las tareas que Poblete realizaba en la institución de Ginebra. En primer lugar, se recomendó al Consejo de Administración de la OIT la realización de diversos estudios sobre las condiciones de trabajo en la agricultura y la situación de la población indígena. En este último caso, se le asignó a Poblete la tarea de llevar a cabo una investigación sobre la condición indígena en el Perú, la cual fue publicada en $1938^{24}$. Uno de los aportes principales de este estudio fue determinar la cantidad de indígenas que habitaban el país y sus modalidades de producción en la agricultura e industria. Además, propuso una serie de medidas legales y educativas, con el fin de evitar las formas de trabajo compulsivo y lo extendido que estaba el uso del alcohol y de la coca entre la población indígena. En segundo lugar, la Conferencia Interamericana del Trabajo fue la instancia para que los delegados expresaran una serie de demandas en orden a acercar aún más las tareas de la OIT al continente, obligando a crear nuevas oficinas de corresponsales, entre ellas una en Santiago de Chile ${ }^{25}$. El responsable de organizar dicha oficina fue Moisés Poblete, el funcionario más calificado de la OIT en materias regionales. Al parecer el traslado a América del Sur, acordado a comienzos de 1937, suponía "proseguir las investigaciones sobre las condiciones de vida y de trabajo de los indígenas en ciertos países y para cumplir otras tareas", como lo confidenció el mismo Poblete en una carta ${ }^{26}$. En ella proponía una estadía de dos años en el cargo de corresponsal en Santiago, pero realizando las mismas funciones en países vecinos. Esta estadía, que se transformó en definitiva le permitió desde Chile continuar con sus responsabilidades de elaborar informes sobre la región y participar en distintas reuniones internacionales, como la Conferencia Interamericana del Trabajo de la Habana de 1939 y la Conferencia Internacional del Trabajo de Nueva York de $1941^{27}$.

${ }^{22}$ Poblete, Moisés, Problemas sociales y económicos de América Latina (Santiago, Universidad de Chile, 1936).

${ }^{23}$ Ibíd., p. 2.

${ }^{24}$ Poblete, Moisés, Condiciones de vida y de trabajo de la población indigena del Perú (Ginebra, OIT, 1938).

${ }^{25}$ Luego de la Conferencia Interamericana del Trabajo de 1936 se crearon, además de Santiago, las corresponsalías de la Habana, Caracas, Montevideo, Lima y Bogotá. Véase OIT, Segunda Conferencia Interamericana del Trabajo. Memoria del Director (Ginebra, OIT, 1939), p. 84.

${ }^{26}$ Carta confidencial de Poblete al director de la OIT, del 1 de febrero de 1937, AOIT, P 2037, M. Poblete Troncoso.

${ }^{27} \mathrm{Su}$ experiencia en esa conferencia es recogida en Poblete, Moisés, La Conferencia 
A su regreso al país se integró a una red de intelectuales que se había formado al alero de la Universidad de Chile. En 1939 ingresó a la Facultad de Derecho de esta Universidad como profesor extraordinario de Economía Social y Legislación de Trabajo. En la época la Facultad de Derecho comprendía un cuerpo variado de profesores y de programas interesados en las problemáticas sociales y laborales, tendencia que seguía aquella de los años 1910 y 1920. Figuras destacadas eran los profesores Francisco Walker Linares, Alfredo Gaete, Héctor Escribar y Carlos Vergara, quienes en la década de 1930 y 1940 promovieron los primeros estudios sobre el movimiento obrero chileno y perspectivas comparadas entre la legislación laboral chilena y la del resto del continente. La presencia de este perfil de profesores con importantes vínculos internacionales, incluso con el trasfondo de la Segunda Guerra Mundial, y el apoyo de la misma Universidad de Chile, fue favorable para el trabajo de Poblete. En 1942 tuvo una destacada participación en la organización de la Primera Conferencia de Seguridad Social realizada en Santiago, publicando para la ocasión un nuevo balance sobre la legislación social del continente americano, el cual incluyó algunos artículos ya publicados y conferencias dictadas entre 1939 y $1940^{28}$.

Es necesario precisar que Poblete compartía con otros intelectuales de su generación, formados en las instancias internacionales, la creencia de que la guerra traería un balance positivo a la hora de juzgar los avances del continente en el ámbito social. Así como la Primera Guerra Mundial había significado la toma de conciencia de la necesidad de promover la paz y la justicia social como valores universales, la Segunda Guerra Mundial -señalaba Poblete- debía promover una nueva sociedad sustentada en la seguridad social y económica.

Para la región, que se había mantenido alejada de la guerra y donde se habían desarrollado diversas reuniones internacionales, existían perspectivas auspiciosas: 'No creemos, sin embargo, que la guerra actual traiga una transformación total en las actuales concepciones y realizaciones económico-sociales de los países hispanoamericanos. Pensamos, eso sí, que la evolución económico-social de América va a acelerarse, y que va a intensificarse, en algunos países y a cimentarse y perfeccionarse en otros, las conquistas sociales ${ }^{22}$.

Según Poblete, frente a un mundo convulsionado como el que ofrecía la Segunda Guerra Mundial había que proponer un programa de cambios sujeto a la planificación y a la evaluación de los expertos.

Un dato ilustrativo dice relación con la posición político-ideológica de Moisés Poblete. Si bien no se le conoce militancia formal en ningún partido, colaboró con la administración de Arturo Alessandri (1920-1925) y siempre tuvo una gran consideración por su obra de gobierno. De tendencia liberal, puede ser considerado un socialdemócrata, apoyando durante su vida un proyecto de cambio social de carácter evolutivo y asignándole al movimiento obrero la labor de educar al pue-

internacional del trabajo de Nueva York: 27 de octubre-6 de noviembre de 1941 (Santiago, Imprenta Universitaria, 1942).

28 Poblete, Moisés, Evolución del Derecho Social en América (Santiago, Editorial Nascimiento, 1942).

${ }^{29}$ Ibíd., p. 16. 
blo, más bien con un perfil técnico y en asociación con los organismos públicos y organizaciones internacionales como la OIT. A diferencia de muchos de su generación, nunca coqueteó con el corporativismo, ideología a la cual le asignaba poca densidad teórica y la situaba cercana al autoritarismo. Por otra parte, si bien no fue un anticomunista, y reconoció al Partido Comunista de Chile como una organización democrática y partícipe del sistema, fue crítico de lo que llamaba "sindicalismo revolucionario", con inclinaciones internacionalistas y permeado por los intereses políticos ${ }^{30}$.

\section{EL DERECHO LABORAL AMERICANO}

Si bien América Latina había dado pasos importantes en la legislación laboral, consagrando incluso constitucionalmente muchos de esos derechos, como el caso de México (1917) y Chile (1925), solo a fines de la década de 1920 se realiza el primer balance sobre el estado de la legislación laboral en América Latina. Dicho balance lo llevó a cabo la OIT, necesitada de tener un catastro general sobre el cumplimiento efectivo de las convenciones internacionales del trabajo ratificadas hasta ese momento, recurriendo para su elaboración a quien se consideraba como el intelectual que mejor conocía la región, el abogado chileno Moisés Poblete. El informe comprendía dos volúmenes. El primero, era un análisis general de la situación legislativa del continente y el segundo un análisis particular de cada país latinoamericano ${ }^{31}$.

El objetivo del informe de 1928 y 1929 no era solamente compilar el progreso del continente en materia social o de ofrecer a la opinión pública de América Latina una síntesis para comparar y evaluar los alcances legislativos. Aunque esos aspectos eran importantes, la naturaleza del esfuerzo se dirigía a otro nivel de desafío. Como el informe había sido elaborado gracias al conjunto de informaciones que los gobiernos y los corresponsales dirigían periódicamente a la OIT, dicha publicación era prueba de las relaciones construidas con el continente. De una o de otra forma, el informe buscaba iniciar una nueva etapa en las relaciones entre la OIT y los países del continente, superando las siguientes dificultades: la ausencia de reconocimiento sobre el papel que la región podía jugar en el universo social del periodo, la incomprensión de las particularidades del continente y la ignorancia que en la OIT se tenía sobre el lugar exacto que América Latina ocupaba en la evolución material y moral del mundo -una de las ideas preferidas de Albert Thomas-. Que el informe haya sido elaborado por Moisés Poblete, uno de los funcionarios de la OIT con mayor reconocimiento, y no por un simple corresponsal, según el procedimiento tradicional, era la demostración de que ese informe no era solo un esfuerzo de compilación para llenar los estantes de las bibliotecas, sino que, por el contrario, para situar al continente como un interlocutor y miembro de pleno derecho de la OIT.

En el balance del informe de Poblete tres aspectos fundamentales se pueden

30 Poblete, Moisés, La organización sindical en Chile y otros estudios sociales (Santiago, Imprenta Ramón Brías, 1926), p. 13.

${ }^{31}$ OIT, Legislación social, cit. (n. 17). 
destacar. En primer lugar, la existencia de una legislación social del todo nueva, que, siguiendo el modelo europeo, se había adaptado a las realidades particulares del continente. En segundo lugar, la identificación de las particularidades propias que habían marcado ese primer esfuerzo legislativo de la región. Por último, la tentativa de reflexionar sobre los puntos en común que la legislación de cada país presentaba y sobre la existencia o no de un derecho social del continente americano.

Si bien el informe reconocía las dificultades que existían en aplicar la legislación social de acuerdo con estándares internacionales, también buscaba responder a las críticas dirigidas a la OIT por la ausencia de colaboración y de acompañamiento en la implementación de esa legislación ${ }^{32}$. Incluso si el informe de Moisés Poblete reconocía en parte esas críticas, el esfuerzo de compilación del progreso legislativo del continente era una prueba concreta de la tentativa de la OIT de dar un lugar de importancia a la región en los avances de la legislación social internacional. A decir del informe, dos aspectos justificaban el retardo en ese esfuerzo de recopilación de la legislación continental. En primer lugar, la legislación latinoamericana era del todo nueva, de algunos años solamente y, en consecuencia, marcada por las vicisitudes propias de una legislación que se parecía más a un conjunto de leyes sobre diferentes aspectos que a un verdadero código laboral moderno. $\mathrm{Al}$ respecto, el informe señalaba: "Debido a lo reciente de la legislación social de los países americanos, no ha podido tener la larga experiencia que en las viejas naciones europeas. Esta circunstancia, forzosamente, ha debido motivar, en primer lugar, defectos justificados en la legislación misma, una gran movilidad en los textos, o sea reformas más o menos continuadas, y, finalmente, dificultades en su aplicación" 33 .

En segundo lugar, era necesario ajustar el esfuerzo de los países de la región por ir más allá de la copia simple de la legislación social europea, lo que explicaba, en parte, la temprana modificación de algunas leyes o algunos problemas de aplicación: "Además, estas Leyes se presentan frecuentemente en forma original. No son exclusivamente codificaciones de las experiencias europeas, impuestas por el desenvolvimiento industrial reciente. Son las más de las veces disposiciones nuevas, debidas al carácter especial de la colonización y de la industria que se ha desarrollado en esos países, alguno de ellos inmensos, y todos de escasa población '34́.

Para la OIT, todo lo anterior explicaba ciertos vacíos de la legislación social que se había aprobado. Por ejemplo, el informe señalaba la reglamentación tardía del contrato de trabajo, donde Chile había sido uno de los primeros países en dictar una ley especial sobre el tema, recién en 1924. La mayoría de las naciones habían puesto un gran acento en reglamentar otro tipo de relaciones laborales, como el sistema de enganche y las bolsas de trabajo. En otro aspecto, el informe ponía en perspectiva la diversidad de las realidades del continente, de tipo económico, social o político, las cuales marcaban la naturaleza de su legislación. La diversidad del paisaje legislativo de América Latina era lo más destacable a los ojos de todo europeo interesado en conocer la legislación social de la región. Sin embargo, para la OIT tal diversidad no debía ocultar los esfuerzos comunes que los países

\footnotetext{
${ }^{32}$ Ibíd., p. III.

33 Ibíd., p. XVI.

${ }^{34}$ Ibíd., p. III.
} 
desarrollaban en el mejoramiento de las condiciones sociales de los trabajadores, así como la comunidad de valores que ellos compartían al momento de justificar esta legislación: "A despecho de las diferencias lingüísticas, trató de asegurarse que todos hablaran la misma lengua social" 35 .

Sin embargo, el informe de Poblete no se limitaba a una constatación genérica de las particularidades regionales que habían marcado la legislación social, sino que también identificaba esas particularidades de manera concreta, lo que permitía explicar las diferencias presentes en relación con la legislación europea, construyendo una tipología o clasificación general que obligaba a pensar la legislación como un conjunto ${ }^{36}$.

Según el informe, tres aspectos propios de la región habrían marcado su legislación social. En primer lugar, la inmigración, la cual era vista como un componente esencial del problema social de América Latina, poniendo de relieve el problema de la movilidad del factor trabajo y la necesidad de adaptar la legislación social a las exigencias de un mercado laboral mucho más diversificado. El progreso en la ratificación de las convenciones sobre la igualdad de trato de los trabajadores extranjeros, así como las normas constitucionales que reconocían la igualdad de derechos entre los trabajadores nacionales y los extranjeros, eran la prueba de la importancia dada al fenómeno de la inmigración.

En segundo lugar, el informe destaca la influencia europea en la primera legislación social. Los lazos entre la región y el viejo continente, así como la superioridad con la cual la realidad europea era presentada, eran razones más que suficientes para buscar en Europa modelos de intervención: "El hecho social europeo ha sido inspirador de la solución del problema social americano. Esto se explica porque la cultura de las naciones americanas es, ante todo, una cultura latina y no anglosajona. La enseñanza universitaria en América Latina, las doctrinas económicas y sociales, han estado siempre inspiradas por métodos europeos de enseñanza y por filósofos, economistas y pensadores de la vieja europea, especialmente de Francia" 37 .

Una última característica de la región es el importante componente agrícola en la estructura productiva y social. Los efectos casi nulos de la legislación laboral en la agricultura se explican tanto por el poder de los agricultores en los parlamentos nacionales como por los esfuerzos por mantener las ventajas comparativas del sector exportador. El informe de la OIT, favorable a este segundo aspecto, señalaba: "Hay una resistencia a la legislación, porque la producción agrícola en América Latina ha sido una producción de exportación, y en consecuencia, de competencia sobre el mercado internacional, competencia que una ley económica induce a los productores a producir a muy bajo precio y evitar todo aumento del costo de la producción '38.

Esas condiciones contribuían a la creación de legislaciones muy diferentes, en

${ }^{35}$ Ibíd., p. IV.

${ }^{36}$ Tal calificación se estableció de acuerdo con los criterios de la Oficina de Basiela, de la Asociación Internacional para la protección legal de los trabajadores: I. Protección obrera internacional. II Protección obrera nacional. III. Problemas generales. Ibíd., p. IV.

${ }^{37}$ Ibíd., p. XVII.

${ }^{38}$ Ibíd., p. XVIII. 
principio en relación con las estructuras productivas nacionales: en los sectores agrícolas esta legislación tenía poca influencia, mientras que en los sectores urbanos mucho más. Según el informe, como el componente industrial y manufacturero no era importante en el conjunto de la economía latinoamericana, la resistencia a la legislación social fue débil por parte del empresariado: "Es reconocido que la legislación social se traduce, más o menos, por una carga a la industria, por el aumento del costo de la producción; como la competencia económica existe débilmente en América Latina, la resistencia a la política social no ha sido tan importante en la primera etapa ${ }^{39}$. Además, el informe quería ofrecer un punto de vista comprensivo de las diferencias presentes en el desarrollo legislativo de la región, llamando a la construcción de un consenso al interior de las elites productivas, yendo más allá de los análisis tradicionales que responsabilizaban a los gobiernos del r e t a r d o de la legislación social.

En síntesis, el informe de la OIT buscaba producir una mirada global en un panorama legislativo diverso, reconociendo en los intentos por coordinar la legislación social de los países y lograr acuerdos para la igualdad en el tratamiento entre los nacionales y extranjeros un esfuerzo concreto de construcción de un derecho social americano. Sobre este punto, el informe mencionaba los diversos congresos regionales que habían tratado el tema: el Congreso de Ciencias Sociales de Tucumán de 1916, el Congreso de Previsión Social de Río de Janeiro de 1923 y, por último, el Congreso de Economía Social de Buenos Aires de $1924^{40}$.

A partir del análisis del informe de Poblete, se puede comprender las exigencias de la OIT durante los años 1920 por conocer las características de las diferentes regiones del mundo, en especial indagar sobre las dificultades que tenían algunos países en participar en las conferencias del trabajo de Ginebra. Este informe tiene también el papel de identificar las dinámicas propias del continente americano, alimentando la imagen de una región rica en desarrollo social, aunque impotente en su capacidad de seguir las disposiciones emanadas de la institución de Ginebra.

Sea como sea, el informe reconocía el dinamismo de la legislación del continente y podía presentarse como el portaestandarte de un naciente regionalismo latinoamericano, reconociendo la obra de acercamiento de los intelectuales y de promoción de lo social en los diferentes congresos continentales. A partir de estos primeros análisis, la OIT podía llegar a nuevas conclusiones en cuanto a reconocer otras vías de promoción de una política social que fuese más allá del sistema de ratificación, como por ejemplo la asistencia técnica en el ámbito de la legislación laboral que ya había sido aprobada ${ }^{41}$.

${ }^{39}$ Ibíd., p. XVII.

40 Para profundizar sobre estos congresos, véase Zimmermann, Eduardo, Los liberales reformistas. La cuestión social en la Argentina (Buenos Aires, Editorial Sudamericana, 1995), p. 95.

${ }^{41}$ La OIT comienza a desarrollar a partir de mediados de los años 1930 una política de asistencia técnica a los países latinoamericanos. Véase, Plata, Véronique, La difusión de las normas internacionales del trabajo en Venezuela (1936-1939): una práctica de cooperación técnica internacional en la OIT, en Herrera, Fabián y Herrera, Patricio (eds.), América latina y la OIT. Redes, cooperación técnica e institucionalidad social (1919-1950) (Morelia, Universidad Michoacana de San Nicolás de Hidalgo, 2011). 
Este informe de 1928 será complementado en 1936 por otra investigación de Poblete, que recoge a manera de compendio la literatura producida en Hispanoamérica sobre economía social, derecho del trabajo y políticas sociales en general $^{42}$. Organizada por países, el compendio bibliográfico ofrece, además, una búsqueda temática por materias. Sobre el conjunto Poblete señala: “Una conclusión interesante puede también deducirse del examen de conjunto del movimiento ideológico de América en los problemas económico-sociales, considerados desde su aspecto jurídico, es la de la formación, cada día más acentuada, de esta nueva modalidad del derecho que constituye lo que muchos han llamado el 'derecho del trabajo' y otros 'el derecho nuevo', como lo designa el eminente profesor argentino Alfredo Palacios ${ }^{\$ 43}$.

Poblete terminaba afirmando que la variedad de la literatura social producida en los países de Hispanoamérica demostraba la solidez que tenía la cultura social en el continente. En especial, la seguridad social -señalaba- había dejado de ser entendida como una serie de medidas poco articuladas para enfrentar los accidentes o algunos riesgos de la vida moderna, para pasar a transformarse en una dimensión que abarcaba todos los aspectos de la existencia.

Por último, es interesante la visión evolutiva que tenía Poblete sobre el avance de la legislación laboral, colocando a Chile como un país referente en cuanto a logros y etapas que las naciones debían pasar para mostrar un progreso social. En este sentido, el abogado chileno ofrece una primera periodificación de los avances en materia de derecho del trabajo, señalando que existían tres etapas plenamente identificadas en esa evolución ${ }^{44}$. El primer periodo, comenzaba a mediados del siglo XIX y finalizaba hacia el 1900, estando bajo la influencia del i n d i v i d u a li s m o e c o n ó m i c o, heredero del economista francés Courcelle-Seneuil y cuyos seguidores chilenos eran Miguel Cruchaga Montt y Zorobabel Rodríguez. El segundo, iba entre 1900 y 1924, caracterizado por algunas leyes de carácter social, "aunque escasas y fragmentarias" ${ }^{2}$. La escasa industrialización y la lenta formación de un movimiento obrero explican, según Poblete, lo limitado de la legislación laboral. Solo cuando se fortalezca el movimiento obrero en torno a una naciente industrialización, se verán las primeras respuestas legislativas para enfrentar la cuestión social. El tercer periodo, comenzaba en 1924 y se extendía hasta la década de 1940, caracterizado por una legislación social más sistemática, los primeros proyectos de código del trabajo, y su posterior codificación en 1931.

\section{LA SEGURIDAD SOCIAL EN CHILE Y AMÉRICA LATINA}

Un aspecto que es importante a destacar del quehacer de Poblete, y que es complementario a su labor recopilatoria de la legislación laboral chilena y continental, dice relación con los estudios sobre la seguridad social. Si bien nunca

${ }^{42}$ Poblete, Ensayo, cit. (n. 19).

${ }^{43}$ Ibíd., p. 3.

${ }_{44}$ PoBlete, Moisés, El derecho del trabajo y la seguridad social en Chile (Santiago, Editorial Jurídica, 1949).

${ }^{45}$ Ibíd., p. 15. 
fue un experto en la materia, su interés por recopilar las investigaciones y legislaciones sobre el tema se explica por el fuerte nexo existente entre los avances de la legislación laboral y las modalidades con que las sociedades enfrentan los riesgos de la vida moderna, en especial los accidentes del trabajo, las enfermedades profesionales y la vejez.

Chile había aprobado en 1924 un pionero sistema de seguros sociales, a través de la Caja del Seguro Obrero (Ley 4054) que hacía obligatorio el seguro de enfermedad, maternidad y vejez para toda persona menor de 65 años que no tuviera otro medio de subsistencia que su salario. El financiamiento era de tipo tripartito, considerando el aporte de trabajadores, empresarios y Estado, siendo exitoso en ofrecer beneficios a quienes participaban de su financiamiento, excluyendo, en cambio, a un porcentaje importante de la población: campesinos, trabajadores informales y niños. Con las reformas de fines de los años 1930 se procuró proveer una atención especializadaa las mujeres y niños que no cubría el seguro social y extender los beneficiosa la familia de los cotizantes, resolviendo el problema evidente de inequidad que había marginado de las prestaciones sociales a estos grupos por no estar integrados al mercado laboral, pero que sufrían los mismos riesgos. Por ejemplo, la ley 6.236 de 1938, conocida como ley madre-niño, estableció el examen médico preventivo de la mujer embarazada y el control periódico del niño hasta los dos años, entregando los medicamentos y los alimentos necesarios. Por otra parte, la ley 6.174 de medicina preventiva del mismo año promovió exámenes médicos periódicos para la población asegurada, con el fin de evitar el aumento de gastos de administración que afectaban a la Caja del Seguro Obrero y a las indemnizaciones de invalidez. Además, la ley garantizabael reposo, la protección del empleo y del salario, hasta la recuperación total del paciente ${ }^{46}$.

Por su parte, la política de la OIT sobre los seguros sociales se había desarrollado a través de una serie de convenciones y recomendaciones aprobadas en el curso de los años 1920 y 1930, y que comprendía aspectos tan variados como accidentes del trabajo, enfermedades profesionales, y el seguro de enfermedad, de vejez, de invalidez y muerte. El amplio consenso obtenido en la aprobación de esas convenciones muestra, por un lado, los acuerdos previos que habían instalado a nivel nacional las elites gobernantes, obreras y patronales sobre el tema, y, por otro, los esfuerzos por avanzar hacia un sistema de seguro obligatorio, descartando la previsión voluntaria, a pesar de las demandas de ciertos sectores.

A partir de los años 1940, la seguridad social toma un lugar importante en los debates sobre una política social moderna, buscando mejorar las condiciones de vida del conjunto de la población y no solamente de los sectores más integrados. Muchas de esas disposiciones habían sido consagradas en las constituciones políticas, como lo veremos más adelante ${ }^{47}$. Además, la noción de seguridad social

46 Labra, María Eugenia, Medicina social en Chile: propuestas y debates (1920-1950), en Cuadernos de Medicina Social, 44 (Santiago, 2004), pp. 207-219.

47 Por ejemplo, se puede citar la constitución de México (1917), Chile (1925), Uruguay (1940), Cuba (1940), Colombia (1945) y Brasil (1946). Véase, De Madariaga, Eduardo, La reforma total de la Constitución: los derechos sociales en las constituciones de América (Buenos Aires, Biblioteca del Congreso de la Nación, 1948). 
se transforma en una herramienta de reconocimiento de todos los sectores que se sienten partícipes de un programa global de política social. Los fundamentos de esta nueva política se encuentran en la Social Security Act del gobierno de Roosevelt, que señala que "la seguridad social debe ser una organización estructural de la sociedad, garantizando a todos los ciudadanos la posibilidad de una vida libre $y$ de pleno desarrollo de sus facultades". Ese concepto de seguridad se consolidó a partir de los estudios de la comisión Beveridge que la definía como la abolición del estado de necesidad, asignándole un papel importante en la política económica de un país, como parte integrante de los programas de pleno empleo y de aumento del consumo general ${ }^{48}$.

Un avance en cuanto a la seguridad social en América Latina fue la convocatoria a la Conferencia Interamericana de Seguridad Social, desarrollada en Santiago de Chile en 1942, resultado de la lenta formación de una red favorable a compartir experiencias en materia de seguros sociales y en la cual participaban gobiernos, agencias privadas y los técnicos de la OIT $^{49}$. Las resoluciones de la Conferencia apuntaron a promover la unificación de los sistemas de seguros sociales, la implantación de un programa de seguros para el trabajador y su familia, así como los trabajadores independientes, agrícolas y domésticos. Además, señaló la necesidad de avanzar en la puesta en práctica de programas de seguros por etapas en aquellos países donde un programa integral de seguros fuese difícil de aplicar. Por otra parte, resolvió la extensión de los seguros a los trabajadores intelectuales y a las profesiones liberales, sobre la base de un programa sistemático y coordinado de seguros de accidentes del trabajo y enfermedades profesionales. Por último, se propuso avanzar en la integración regional de los sistemas de seguros sociales, en especial en cuanto a la unificación de la información bioestadística para que sea posible su comparación internacional.

Es en este contexto de debates sobre la seguridad social que Moisés Poblete publica en 1942 su libro Evolución del Derecho Social en América ${ }^{50}$. Sin embargo, es necesario destacar que su interés por la seguridad social provenía de mediados de los años 1920, cuando publicó Instituciones de previsión social, un trabajo de compilación de los diversos regímenes previsionales existentes ${ }^{51}$. La publicación de 1942 sigue el mismo derrotero intelectual de sus anteriores publicaciones, presentando en este libro una visión panorámica de cómo había evolucionado el Derecho Social en América Latina entre 1930 y 1940, destacando dos aspectos novedosos, los que no escaparon al ojo minucioso de Poblete: el contenido social de las nuevas constituciones del continente americano y las instancias de cooperación internacional que se habían dado en la región desde mediados de los años 1920 .

Sobre el primer punto, el abogado chileno destaca el contenido social de las

48 Para una vision general, véase Heidenheimer, Flora y Arnold, The Development of Welfare State in Europe and America (New Jersey, Brunswick, 2009).

49 Conferencia Interamericana de Seguridad Social, Actas de la Primera Conferencia interamericana de Seguridad Social (Santiago, La Conferencia 1943).

${ }^{50}$ Poblete, Moisés, Evolución del Derecho Social en América (Santiago, Editorial Nascimento, 1942).

51 Poblete, Moisés, Instituciones de previsión social (Santiago, Imprenta Recurba, 1927). 
constituciones políticas del continente, lo que supone que el Estado se compromete en proteger al obrero, reglamentando sus condiciones de trabajo y un mínimo de protección social. En especial se habla de la limitación de la jornada de trabajo, el descanso dominical, participación en los beneficios y las vacaciones pagadas, lo que habla de una evolución progresiva en el reconocimiento de derechos desde los aspectos clásicos de las condiciones de trabajo a los aspectos vinculados al ocio y el tiempo libre. En el caso de América Latina, se destaca la Constitución mexicana de 1917, chilena de 1925, peruana de 1933, venezolana de 1936 y cubana de 1940.

Sobre el segundo aspecto, las manifestaciones de cooperación entre los países del continente y la OIT, Poblete reconoce los esfuerzos realizados en orden a convocar instancias de intercambio de experiencias y de cooperación con el fin de avanzar en un derecho social americano. Para ello hace referencia a un sinnúmero de congresos, seminarios y conferencias realizadas entre 1916 y 1940 en América Latina, las cuales se sintetizan en las siguientes: Congreso de Tucumán (1916); Congreso de Previsión Social de Río de Janeiro (1923); Congreso de Economía Social de Buenos Aires (1924); Primera Conferencia Interamericana del Trabajo de Santiago (1936); Primer Congreso Panamericano de la Vivienda Popular (1939); Segunda Conferencia Interamericana del Trabajo de Cuba (1939); Tercera Conferencia Internacional de Alimentación de Buenos Aires (1939); Primer Congreso Indigenista Interamericano de Pátzcuaro (1940).

Sin embargo, Poblete era consciente de las dificultades en consolidar los avances legislativos y asegurar un clima de opinión favorable a la intervención del Estado y el respeto de derechos sociales fundamentales. En 1949 señalaba lo siguiente, con palabras que resuenan muy actuales: "Limitados sectores de la opinión pública de nuestros países, pero que cuentan con medios de difusión de sus ideas más amplios en la prensa, critican y analizan desde sus limitados puntos de vista lo que ellos califican de una carga excesiva de esta protección jurídico-social del trabajador; agregan que esta estructura social suprime o anula la iniciativa individual, etc. Pero esos sectores y sus intérpretes olvidan que el progreso económico no puede realizarse armónicamente sin una protección adecuada del elemento humano, que lo ponga a cubierto de los riesgos profesionales y sociales; que vele por sus derechos inalienables ${ }^{\text {} 2}$.

Terminaba señalando que el mejoramiento del estándar de vida no podía hacerse realidad solo a través del aumento de la producción: "es necesario un progreso paralelo del derecho del trabajo y de la seguridad social" 53 .

La labor profesional de Poblete seguirá activa durante los años 1950 y 1960, ampliando sus estudios hacia los estándares de vida, la reforma agraria y la demografía, lo que le permitió seguir siendo protagonista del debate académico hasta su muerte en 1970.

\footnotetext{
${ }^{52}$ Ibíd., p. 10.

53 Ibíd.
} 


\section{CONCLUSIONes}

El presente artículo ha querido relevar la persona y obra del abogado chileno Moisés Poblete Troncoso. La escasez de estudios sobre su obra, así como la ausencia de citaciones sistemáticas en las investigaciones sobre legislación laboral, movimiento obrero y seguridad social, dan cuenta de cierto desconocimiento de las contribuciones que hizo sobre diversas materias. Lo primero que se destaca en su recorrido profesional es su papel importante en la naciente legislación laboral chilena y cómo su experiencia y contactos forjados como director de la Oficina del Trabajo entre 1920 y 1925, le permitieron forjar vínculos con directores de otras oficinas de igual naturaleza del continente, así como con otros intelectuales, políticos y dirigentes sindicales. Como se ha querido destacar en este artículo, lo interesante de la obra de Poblete es el sentido de actualidad que tienen sus trabajos, los cuales se desarrollaron al alero de la función pública y vida universitaria, lo que le permitió discutir sobre la legislación laboral en el momento mismo que eclosionaba la llamada cuestión social.

La postura reconocida en sus escritos, cercana a la social democracia europea y de lo que en la época se denominaba liberalismo social, le permitió reconocer en la legislación laboral el camino correcto en la construcción de una sociedad de derechos, evitando las soluciones violentas planteadas por los sectores más radicales, así como las actitudes de negación de la cuestión social propuesta por ciertos sectores conservadores.

Su ingreso como funcionario internacional a la OIT en 1927, es prueba de sus competencias y el desarrollo de su perfil internacional, lo que le permitió transformarse en un intelectual de primer orden y el que más conocía sobre legislación social del continente americano. Este paso por la institución de Ginebra le permitió multiplicar sus contactos y viajar en diversas ocasiones por el continente, lo que ayudó en la construcción de una obra de alcances supra nacionales.

Por último, si bien su obra puede ser caracterizada de ecléctica y en extremo variada, lo que le permitió hacer aportes en áreas tan diversas como legislación laboral, organización sindical, estándares de vida, consumo, organizaciones internacionales, organismos técnicos del trabajo, tiempo libre, derechos humanos, entre otros, es posible identificar un hilo conductor. Para Moisés Poblete, los avances en la legislación laboral y seguridad social eran, por una parte, el marco propicio para insertar internacionalmente a los países latinoamericanos en el proceso civilizatorio y que hiciera posible, por otra, la existencia de un mundo que marche por el camino de la paz y la justicia social.

\section{BiBLIOGRAFÍA}

Fuentes de Archivo

Archivo de la Oit, Expedientes personales, P 2037. Moisés Poblete Troncoso.

Archivo Nacional, Dirección General del Trabajo, Vol. 76, Comunicaciones enviadas, 1921. 
Referencias bibliográficas

Conferencia Interamericana de Seguridad Social, Actas de la Primera Conferencia interamericana de Seguridad Social (Santiago, La Conferencia 1943).

De Madariaga, Eduardo, La reforma total de la Constitución: los derechos sociales en las constituciones de América (Buenos aires, Biblioteca del Congreso de la Nación, 1948).

Feliú Cruz, Guillermo, Moisés Poblete Troncoso: la bibliografía de las ciencias sociales (Santiago, Biblioteca Nacional, 1970).

Heidenheimer, Flora y Arnold, The Development of Welfare State in Europe and America (New Jersey, Brunswick, 2009).

Herrera, Fabián y Wehrl, Yannick, Le Bureau international du Travail et l'Amérique latine durant l'entre-deux-guerres: problèmes et enjeux, en LESPINET-MORET, Isabelle y VIET, Vincent, L'Organisation internationale du Travail. Origine, développement et avenir (Rennes, PUR, 2011).

Herrera, Patricio, La Confederación de Trabajadores de América Latina en la historiografía obrera.1938-1963, en Cuadernos de Historia, 36 (Santiago, 2012), pp. 85-117.

Labra, María Eugenia, Medicina social en Chile: propuestas y debates (1920-1950), en Cuadernos de Medicina Social, 44 (Santiago, 2004), pp. 207-219.

Morris, James, Las elites, los intelectuales y el consenso: estudio de la cuestión social y del sistema de relaciones laborales (Santiago, Editorial del Pacífico, 1967).

OIT, La legislación social de América Latina (Ginebra, OIT, 1928-1929), vol. 1-2.

OIT, Segunda Conferencia Interamericana del Trabajo. Memoria del Director (Ginebra, OIT, 1939).

Poblete, Moisés y Álvarez, Oscar, La legislación social obrera chilena (recopilación de leyes y disposiciones vigentes sobre el trabajo y la previsión social (Santiago, Imprenta, 1924).

POBLETE, Moisés, Legislación sobre hijos ilegitimos (cuestión social) (Santiago, Imprenta El Progreso, 1912).

-El balance de nuestro seudo régimen parlamentario (Santiago, Editorial Numen, 1920).

-La organización sindical en Chile y otros estudios sociales (Santiago, Imprenta Ramón Brias, 1926).

-Instituciones de previsión social (Santiago, Imprenta Recurba, 1927).

- La legislación social de América Latina (Ginebra, OIT, 1928-1929), vol. 1-2.

-Ensayo de bibliografía social de los países hispanoamericanos (Santiago, La Nación, 1936).

-Problemas sociales y económicos de América Latina (Santiago, Universidad de Chile, 1936).

-Condiciones de vida y de trabajo de la población indígena del Perú (Ginebra, OIT, 1938).

-La Conferencia internacional del trabajo de Nueva York: 27 de octubre-6 de noviembre de 1941 (Santiago, Imprenta Universitaria, 1942).

-Evolución del Derecho Social en América (Santiago, Editorial Nascimento, 1942).

-El movimiento obrero latinoamericano (México, FCE, 1946).

- El derecho del trabajo y la seguridad social en Chile (Santiago, Editorial Jurídica, 1949).

Plata, Véronique, La difusión de las normas internacionales del trabajo en Venezuela (1936-1939): una práctica de cooperación técnica internacional en la OIT, en HERRERA, Fabián y Herrera, Patricio (eds.), América latina y la OIT. Redes, cooperación técnica e institucionalidad social (1919-1950), (Morelia, Universidad Michoacana de San Nicolás de Hidalgo, 2011). 
Suriano, Juan, El Departamento Nacional del Trabajo y la politica laboral durante el primer gobierno de Hipólito Yrigoyen, en Ben Plotkin, Mariano y Zimmermann, Eduardo (compiladores), Los saberes del Estado (Buenos Aires, Edhasa, 2012).

YẤNEZ, Juan Carlos, La intervención social en Chile (1907-1932) (Santiago, Ril Editores, 2008).

-La OIT en América del Sur. El comunismo y los trabajadores chilenos (1922-1932) (Santiago, Editorial UAH, 2016).

-Moisés Poblete Troncoso y la primera 'Historia del movimiento obrero latinoamericano' (1946), en Izquierdas, 37 (Santiago, 2017), pp. 261-280.

Zimmermann, Eduardo, Los liberales reformistas. La cuestión social en la Argentina (Buenos Aires, Editorial Sudamericana, 1995). 